\title{
An Ambient Intelligent Framework for Residential Monitoring of Dementia Patients using Fuzzy Agents
}

\author{
Faiyaz Doctor \\ Fac. of Engineering \& Computing \\ Coventry University \\ Priory Street \\ Coventry CV1 5FB \\ United Kingdom \\ f.doctor@coventry.ac.uk
}

\author{
Rahat lqbal \\ Fac. of Engineering \& Computing \\ Coventry University \\ Priory Street \\ Coventry CV1 5FB \\ United Kingdom \\ r.iqbal@coventry.ac.uk
}

\author{
Raouf N. G. Naguib \\ BIOCORE \\ Coventry University \\ Priory Street \\ Coventry CV1 5FB \\ United Kingdom \\ r.naguib@coventry.ac.uk
}

\begin{abstract}
In this paper an ambient intelligent-based framework is proposed for the monitoring of dementia patients living in their own homes. Within this framework groups of unobtrusive wireless sensor devices can be deployed at specific locations within a patient's home and accessed via standardized interfaces provided through an open middleware platform. For each sensor group intelligent agents are used to learn fuzzy rules, which model the patient's habitual behaviours in the environment. An online rule adaptation technique is applied to facilitate short-term tuning of the learnt behaviours, and long-term tracking of behaviour changes which could be due to the effects of cognitive decline caused from dementia. The proposed system reports behaviour changes to care providers to enable them to make better-informed assessments of the patient's cognitive abilities and changing care needs.
\end{abstract}

Keywords: Ambient Intelligence, Dementia Care, Fuzzy Logic, Intelligent Agents, Ubiquitous Computing

\section{INTRODUCTION}

Ubiquitous and pervasive computing is a paradigm in which computing technology becomes virtually invisible by being embedded in our environments. As embedded computers get smaller and become integrated into noncompeting artifacts, they can effectively physically disappear. With the addition of communication capabilities and pervasive networking, such artifacts can be associated together and remotely accessed in both familiar and novel arrangements to make highly personalized systems (Doctor-2005). Ambient intelligence $(\mathrm{Aml})$ is a new information paradigm, which combines pervasive computing with intelligent data representation and learning systems. Aml enables the environment to be "aware" of the presence and context of inhabitance, their activities and preferences using transparent, unobtrusive intelligent, personalized interconnected systems and services (Ducatel-2003). The health and social care domain is an important area where pervasive computing and ambient intelligence research are being increasingly applied to provide eHealth related services for our ageing population (Chiarugi-2006). An increase in life expectancy has produced an older population that needs continuous monitoring and assistance, especially in case of progressive age-related illnesses such as dementia.

Dementia is a collective term used to describe various different brain disorders that have in common a loss of brain function that is usually progressive and eventually severe (Alzheimer's Society-2011). The observable characteristics of dementia-related cognitive decline is generally: gradual loss of memory, difficulty in performing familiar tasks or complex tasks, changes in mood or behaviour and disorientation. In the UK there is a gradual increase in the number of elderly people aged 65 and above who suffer from dementiarelated cognitive disorders. The number of patients with dementia in the UK is estimated to be 821,884 , representing $1.3 \%$ of the UK population (Dementia 2010). The majority of dementia sufferers tend to be supported by carers and live independently in their own homes. Due to the progressive and degenerative nature of diseases such as Alzheimer's, long-term remote management of patients is essential in order to track the stages of the disease, adjust the course of therapy and understand the changing care needs of the patient. 
Observing a patient's activities can be used to assess stages of the disease. For example, early stages of cognitive impairment may cause forgetting certain steps in familiar tasks or difficulty in performing new and complex tasks. Whereas in moderate stages the patient may exhibit a lack of awareness in the time of day leading to pronounced changes in behaviour from day to day, or impaired communication (PDE-3193-2004).

In this paper, we propose a framework for an ambient intelligent system which could be deployed in a home-based environment to monitor and track the disease progression of dementia patients, see Figure 1. Our approach would create a Personalized Home Sensor Network (PHSN) for monitoring key aspects of a patient's cognitive behaviour and activities. The PHSN would consist of Local Sensor Groups (LSG) of simple unobtrusive wireless sensor devices, which would be retrofitted to positions and items at locations around the home, and accessed using an open middleware component based software architecture. The placement of each LSG would be determined by a variety of patient-related factors alongside the advice of care staff and health professionals. For each LSG we propose using an intelligent agent learning and adaptation technique that learns fuzzy rules modelling the particularbehaviours of the patient in the environment. The fuzzy rules can be adapted online and used to track medium- to long-term behaviour changes, which could be due to patient's cognitive decline.

In section 2 we present a literature review discussing environmental sensors and wireless sensor network technologies, intelligent agents and fuzzy systems for realising Aml and previous work on the development of assistive technologies for monitoring dementia patients. In section 3 we describe our fuzzy learning and online adaptation approach for the intelligent agent to enable the tracking of cognitive decline in dementia patients. Finally conclusions and future work are presented in Section 4

\section{LITERATURE REVIEW}

Research has shown that people who develop cognitive decline seem to experience subtle changes in daily activities before the diagnosis of dementia is confirmed (Gustafsson-1995). However periodic observations and assessments have been found to be ineffective in detecting or diagnosing cognitive impairment in early stages of the disease (Binegar-2009). Therefore it is important to continually monitor everyday activities over time in order to provide a more effective way of identifying activity changes that can signify cognitive deterioration.
It can be difficult for relatives and carers to continually observe a patient and recognise the subtle changes in a patient's behaviour and daily activities, which may signal progressively worsening stages of the disease. It can also be difficult for health professionals to accurately track the patterns of a patient's cognitive impairment and the impact this is having on daily living and wellbeing.

Wireless sensor networks comprise of clusters of sensor devices equipped with a radio transceiver, and small microprocessor deployed over a specific area, which wirelessly communicate data to a central system. They are based on emerging technologies such as communication technologies (RF communication, ad hoc networking routing), semiconductor, embedded systems and micro sensor technologies [PureLink Technology-2010]. The prevalence of relatively low cost off-the-shelf pervasive wireless sensor devices can be used for sensing motion, light level, temperature, occupancy, chair / bed pressure or appliance hazards such as checking if the cooker has been left on (Woolham-2007). Simple switch sensors can be used to determine if drawers and cupboards have been opened / closed, and whether specific electronic devices have been switched on or off. Radio Frequency Identification (RFID) tagging of daily personal items can be used to track how the patient interacts with and places movable items in the home during routine activities. For the purpose of this paper we will divide these sensors into passive input sensors such as temperature, light level or occupancy, and active output actuation based sensors such as appliance states, sensors for light switches or doors and cupboards states and RFID tracking of movable objects (Matic-2008).

The vast majority of wireless sensor devices will support one of several commercial wireless sensor networking standards such as Zigbee, ANT, ZWave, ONE-NET to name a few. The development of open middleware architectures such as OSGi (OSGi-2011) can be used to dynamically integrate heterogeneous commercial proprietary sensor devices and network technologies together for easy deployment in a residential environment. This can allow Aml systems to seamlessly record data simultaneously from various sensor devices in context of the resident's interactions with the environment (Conde-2010). The OSGI framework can provide a service oriented interface mechanism with which each intelligent agent can homogeneously capture data received from different heterogeneous sensor devices configured within each LSG.

Intelligent agents can be embedded in the user's environment to realize $\mathrm{Aml}$ systems, which can recognize the patient's interactions and program 
themselves to learn their behaviours (Doctor-2005). Soft computing approaches can be used to identify and model behaviours based on the patterns of association between sensed environmental conditions (light level, occupancy, time of day etc) and specific actions being performed by the patient (switching on the lights, changing channels on the TV or moving an object to a specified location). In particular Fuzzy Logic Systems (FLSs) have been credited with being an adequate methodology for designing robust systems that are able to deliver a satisfactory performance when contending with the uncertainty and imprecision attributed to real world pervasive computing environments. An FLS represents a nonlinear mapping of input states to specific output conditions. FLSs provide transparent and flexible model representations which can be generated from data, and are based on linguistic quantifiers and human readable rules. Fuzzy sets provide a methodology for computing with words using linguistic quantifiers such as 'High' or 'Near' (Mendel-2001). These are combined with fuzzy rules which can approximate independent local models of a patient's particular behaviours that map sets of input sensor states to sets of output user action states. The flexibility of fuzzy rule based systems means that individual rules can be adapted to approximate short-term changes in the patient's behaviours and identify medium- to long-term behaviour variations which may be the consequence of progressive cognitive decline.

The importance of pervasive continuous and reliable long-term monitoring of dementia is emphasized in (Arnrich-2010). Significant results have been obtained in the development of smart environments for monitoring hand washing (Peters2009) (Mihailidis-2004), cooking (Mynatt-2004), taking medications (Hayes-2006), dressing activities (Matic-2008), as well as behaviours such as agitation (Biswas-2006) pain and discomfort (Bonroy-2008). These approaches however tend to focus on very specific activities. They relay heavily on the use of cameras and computer vision in detecting and classifying activity patterns, and have tended to be conducted under very controlled settings over short periods of time. Such systems may not necessarily be practical to use in a patient's home due to logistical and privacy related reasons. A study was undertaken on the use of simple motion sensors around the home to spot subtle changes in mobility and behaviour of Alzheimer's suffers over time that may precede memory loss (Neergaard-2007). However this study was again limited to using a small set of sensory inputs which focused on one indicator of cognitive decline.

\section{FUZZY AGENT APPROACH FOR DEMENTIA MONITORING}

The placement of the sensors selected for each LSG would be based on factors such as the physical layout of the residence, the aspects of a patient's cognitive behaviours that need to be monitored, how advanced their dementia already is, and the kind of unassisted activities the patient is able to currently perform. For each LSG the intelligent agent learns and adapts to the user behaviour in the environment using a variation of our previously developed Adaptive Online Fuzzy Inference System (AOFIS) approach described in (Doctor-2005). Our approach is an unsupervised data-driven approach for generating a FLS from data collected through monitoring the patient's interactions with the LSG over a period of time. The generated FLS models the patient's habitual behaviours using fuzzy rules that map sets of input sensor states to sensed output states of devices and utilities based on the patient's interactions with them. The adaptive FLS will passively compare its learning model with the user interactions in the environment. The system will allow the short-term tuning of the learnt fuzzy rules to better approximate the patient's behaviours. Over the medium- to long-term, the agent will record subtle persistent changes in the patient's behaviour as new and adapted fuzzy rules. These can be compared with original tuned behaviour rules to determine how specific behaviours have changed over time in each LSG. The agent can present behaviour changes to health professionals who can make an informed assessment of the cognitive state of the patient. Our approach comprises of five stages as follows (and illustrated in Figure. 1).

\subsection{Capturing Input/Output Data}

The agent passively monitors the user's states and actions within the LSG. Either periodically or when the user performs an action in the environment, the agent will record a 'snapshot' of the current inputs (passive sensor states) and outputs (active sensor states adjusted or previously set by the patient). These 'snapshots' are accumulated over a period of time so that the agent observes as much of the patient's interactions within the environment as possible. The agent learns a descriptive model of the patient's behaviours from the accumulated data. Therefore, given a set of multi-input multioutput data pairs:

$$
\left(x^{(t)} ; y^{(t)}\right), t=1,2, \ldots, N
$$

where $N$ is the number of data instances, $x^{(t)} \in R^{n}$ and $y^{(t)} \in R^{k}$. The agent extracts rules which describe how the $k$ output variables 
$y=\left(y_{1}, \ldots, y_{k}\right)$ are influenced by the $n$ input variables $x=\left(x_{1}, \ldots, x_{n}\right) \in R^{n}$ based on the sampled data.

\subsection{Generation of FLS Modelling Patient Behaviours}

It is necessary to be able to categorise the accumulated user input/output data into a set of fuzzy membership functions which quantify the raw crisp values of the passive input and active output sensors into linguistic labels such as 'normal', 'cold' or 'hot'. As we want to learn the particular behaviours of the user, the agent should define the membership functions from the user's recorded input/output data. In our previous work, (Doctor2005) we developed a technique for generating type-1 membership functions from data that was based on using a Double Clustering approach combining Fuzzy-C-Means (FCM) and agglomerative hierarchical clustering (Castellano2002). We can equally also use FCM over each dimension of the input and output space to generate approximate membership functions (MFs) directly from the fuzzy partition matrix generated by the algorithm.

The defined set of membership functions are combined with the existing user input/output data to extract the rules defining the patient's behaviours. The fuzzy rule extraction approach used is based on an enhanced version of the Mendel Wang (MW) method developed by Wang (Wang-2003). This is a one pass technique for extracting fuzzy rules from the sampled data. The fuzzy sets for the antecedents and consequents of the rules divide the input and output space into fuzzy regions.

The approach extracts multi-input multi-output rules which describe the relationship between $y=\left(y_{1}, . ., y_{k}\right)$ and $x=\left(x_{1}, \ldots, x_{n}\right)^{T}$, and take the following form:

IF $x_{1}$ is $A_{1}^{(l)} \ldots$ and $x_{n}$ is $A_{n}^{(l)}$, THEN $y_{1}$ is $B_{1}^{(l)} \ldots$ and $y_{k}$ is $B_{k}^{(l)}$

$l=1,2, \ldots, M$, where $M$ is the number of rules and $l$ is the index of the rules. There are $V_{n}$ fuzzy sets $A_{s}^{q}, q=1, \ldots, V_{n}$, defined for each input $x_{s}$ where $(s=1, \ldots, n)$. There are $W_{k}$ fuzzy sets $B_{c}^{h}, h=1, \ldots, W_{k}$, defined for each output $y_{c}$ where $(c=1, \ldots, k)$. The agent now extracts rules in the form of Equation (2) from the data. Once the agent has extracted the membership functions and the set of rules from the user input/output data, it has then learnt the FLS that captures the patient's LSG based behaviours. The extracted fuzzy rules represent local models that map a set of inputs to the set of outputs without the need for formulating any mathematical model. Individual rules can therefore be adapted online influencing only specific parts of the descriptive model learnt by the agent (Doctor-2005).

\subsection{Passive Monitoring and Short-Term Online Tuning of Behaviour Rules}

The FLS will passively monitor the patient's behaviours associated with their interactions with the LSG. Rules whose antecedent fuzzy sets match the current sensed state of the environment will be fired and each rule will also keep a frequency of the number of times it has fired over its lifetime. This can be used to prune rules that have fired infrequently and therefore contributed the least to the patient's learnt behaviour model. This will allow the rule base to be kept to a manageable size reducing memory overheads. We also aim to record high frequency associations between groups of firing rules. This would indicate rule groupings that tend to be activated together and therefore associated with the same behaviour.

The patient may make small adjustments to their behaviours within the LSG and the FLS will need to be tuned over a short period of time to allow the system to better approximate the patient's behaviours. Whenever the patient performs an action within the environment (e.g., switching the TV on/off), the agent will check its learnt behaviour rules online and either adapt or add new rules if the patient's action(s) have changed. A snapshot $t$ of the state of the input/output sensors in the environment is recorded and passed to the rule adaptation routine. Each input sensor state is compared to each of the antecedent sets $A_{s}^{(l)}$ of a given rule in the rule base to determine its membership values. The weight $w i^{(l)}$ of the rule is then calculated to determine the degree of firing of the rule as shown in Equation (3).

$$
w i^{\left(t^{\prime}\right)}=\prod_{s=1}^{n} \mu_{A_{s}^{q}}\left(x_{s}\left(t^{\prime}\right)\right)
$$

where the weight of a rule $w i^{\left(t^{t}\right)}$ is a measure of the strength of the points $x^{\left(t^{t}\right)}$ belonging to the fuzzy region covered by the rule. If the weight of the rule is $>0$, then the rule has fired and matched the sensed input states of the environment when the actuation occurred.

The consequent fuzzy sets that give the highest membership to each of the current output sensor states are selected as follows: 


$$
\mu_{B_{c}{ }^{l^{*}}}\left(y_{c}\right) \geq \mu_{B_{c}{ }^{h}}\left(y_{c}\right)
$$

for $h=1,2, \ldots, W$. The $B_{c}$ is chosen as $B^{h^{*}}$. Where $\mathrm{C}=1,2 . ., \mathrm{k}$.

These are compared to the existing consequent sets of the fired rule(s) to determine if there has been a change in the patient's actions (output states). If a change has occurred the new consequent fuzzy sets $B_{c}^{h^{*}}$ will replace the consequent sets of the fired rule(s), adapting the rule(s) to reflect the patient's behaviour changes.

If none of the existing rules fired, new rules are added based on forming rules from the input fuzzy sets. For each input sensor state $x_{s}$ the fuzzy sets that give a membership value where $\mu_{A_{s}^{q}}\left(x_{s}^{\left(t^{\prime}\right)}\right)>t r$ where $t r$ is a firing threshold, are identified. This leads to a grid of identified fuzzy set(s) for each input parameter. New rules can be constructed based on each unique combination of consecutive input fuzzy sets. The consequent fuzzy sets for each of the new rules are determined using Equation (4). This allows new rules to be gradually added to the rule base. The agent will also add new rules when the currently monitored environmental state is undefined by the existing rules in the rule base; i.e. none of the existing rules fired. In this case the agent will create new rules where the antecedent sets reflect the current input states of the environment and the consequent fuzzy sets are based on the current state of the outputs. As each rule represents a local model of a specific patient behaviour, adapting the rule will only change a specific part of the descriptive model learnt by the agent without requiring the remodelling of all the patient's learnt behaviours.

\subsection{Long-Term Rule Adaptation and Generation}

The agent can adapt existing rules and add new rules based on long-term changes in the patient's behaviour within the LSG that may be a direct result of the progressive stages of dementia. The mechanism used to adapt or generate new rules is the same as in section 4.3. The difference here is that rule changes and rule additions will be recorded as new rules in a separate 'probationary' rule base and hence will not change the current behaviour model. As in section 4.3 the individual frequency and high frequency associations of fired rules for both the tuned model rules and the new probationary rules will be recorded. Probationary rule(s) that indicate a persistence and relevant behaviour change over time will then be compared, (on the basis of their antecedent and consequent fuzzy sets), to the current LSG behaviour rules to determine which behaviours have been changed.

These long-term rule changes will then be fed back to update the patient learnt behaviours rules, allowing the system to adapt its behaviour model to account for how the patient's decline in their cognitive abilities is affecting daily activities over time. It is conceivable that over very long periods of time patient's behaviour models may need to be rebuilt from monitoring the patient interactions again. This is likely to occur due to significant changes in the patient's daily routines, or reconfiguring the LSG by adding or removing sensor devices.

Because our framework decomposes the pervasive environment into units of LSGs, it allows each agent-based behaviour model to be associated with a specific sensing area in the home, which can be individually monitored to track the patient's cognitive state. Each behaviour model is also more compact due to smaller more manageable input sizes and hence tractable in terms of the size of the generated rule base. This also means that the fuzzy behaviour rules are more easily interpretable to end users of the system.

\subsection{Assessment of LSGs Behaviour Changes}

For each LSG, behaviour changes will be reported to health professionals and care providers in the form of linguistically interpretable rule comparison reports outlining behaviour changes that have taken place. This will allow observers to make an informed assessment of whether or not the patient's condition has worsened due to cognitive decline, their state of mind and ability to perform routine activities in their home. This will enable a judgment to be made on how their care plan needs to be updated to address changes in their care needs. 


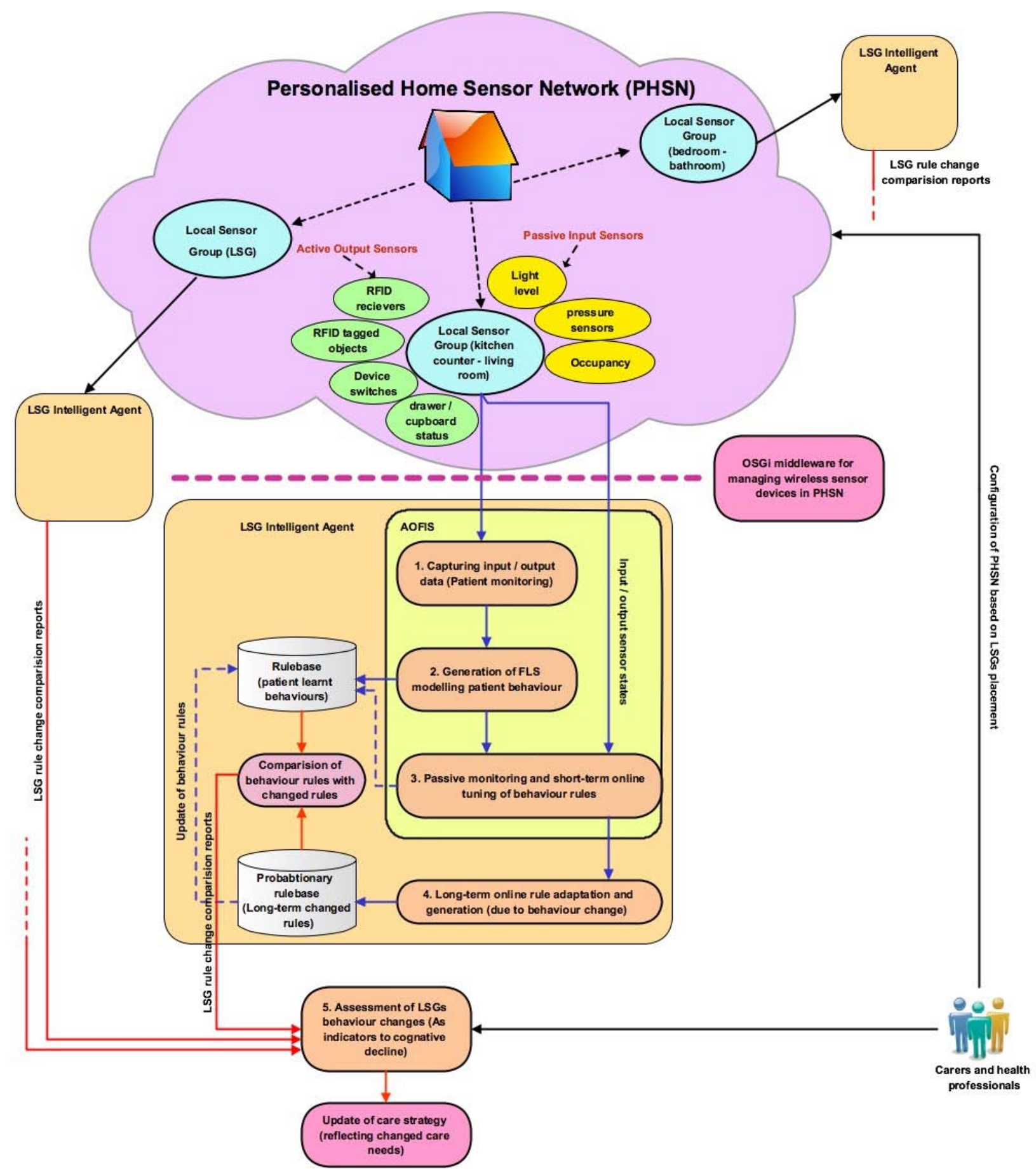

Figure 1: Flow diagram showing the proposed Aml framework comprising of the PHSN and the intelligent fuzzy agent learning and adaptation stages for a given LSG.

\section{CONCLUSION}

In this paper, we propose a framework for an ambient intelligent system to monitor and track the disease progression of dementia patients. The system could be deployed in a patient's home environment where sensors are placed in grouped units pertaining to specific locations and factors related to the patient's disease severity and the opinions of care providers. We suggest the use of a middleware architecture for providing standardized interfaces for communicating with various proprietary wireless sensor devices. For each LSG we propose using an intelligent agent employing a fuzzy learning and adaptation technique for generating fuzzy rules that model the normal habitual behaviours of the patient in the environment. The fuzzy rules can be adapted online and used to track medium- to long-term behaviour changes, which could be due to patient's cognitive decline. These changes are presented to care providers to enable them to make more accurate assessments of the patient's cognitive 
abilities in performing daily tasks, and the kind of additional care support the patient needs. We have demonstrated through previously conducted experiments in a real pervasive computing environment, that our intelligent agent approach can learn to model the user's behaviours and provide short-term tuning of the model to better approximate the learnt behaviours.

For our current and future work we intend to carry out experiments to test the stability and robustness of our approach based on long-term monitoring of users and its ability to detect and report subtle long-term behaviour changes based on the user's activity variations that would simulate effects of dementia-related cognitive decline. We eventually aim to deploy a prototype system within a residential care setting to monitor a patient with dementia to evaluate the system's ability in providing insight into long-term behaviour variation to help care providers assess the changing care needs of the patient.

\section{REFERENCES}

Alzheimer's Socity (2011) About Dementia. http://alzheimers.org.uk/site/scripts/documents.php ?categoryID=200120, 30 June 2011.

Arnrich, B. Mayora, O. Bardram, J., and Troster, G. (2010) Pervasive Healthcare: Paving the Way for a Pervasive, User-Centered and Preventive Healthcare Model, Methods of Information in Medicine, 49, 1 67-73.

Binegar, D. L. Hynan, L. S. Lacritz, L. H. Weiner, M. F., and Cullum, M. C. (2009) Can a Direct IADL Measure Detect Deficits in Persons with $\mathrm{MCl}$ ? Current Alzheimer Research, 6, 1, 48-51.

Biswas, J. et al. (2006) Agitation Monitoring of Persons with Dementia based on Acoustic Sensors, Pressure Sensors and Ultrasound Sensors: A Feasibility Study, In Proceedings of The International Conference on Aging, Disability and Independence, St. Petersburg, Florida, 1-5 February, pp. 3-15, Amsterdam, IOS Press.

Bonroy, B. et al (2008) Image Acquisition System to Monitor Discomfort in Demented Elderly Patients", In Proceedings of the $18^{\text {th }}$ ProRISC Annual Workshop on Circuits, Systems and Signal Processing, Veldhoven, The Netherlands, 29-30 November.

Castellano, G. Fanelli, A. M., and Mencar, C. (2002) Generation of Interpretable Fuzzy Granules by a Double-Clustering Technique, Archives of Control Sciences, 12, 4, 397-410.
Chiarugi, F. Zacharioudakis, G. Tsiknakis, M. Thestrup, J. Hansen, K. M. Antolin, P. Melgosa, J. C. Rosengren, P., and Meadows, J. (2006) Ambient Intelligence support for tomorrow's Health Care: Scenario-based requirements and architectural specifications of the EU-Domain platform. In Proceedings of the International Special Topic Conference on Informational Technology in BioMedicine, loannina, Greece, 2628 October.

Conde, D. Ortigosa, J. M. Javier, F. and Salinas, J. R (2010) Open OSGi Middleware to Integrate Wireless Sensor Devices into Ambient Assisted Living Environments. In Proceedings of AALIANCE Conference, Malaga, Spain, 11-12 March.

Dementia 2010 (2010) Health Economics Research Centre, University of Oxford, Oxford, UK.

Doctor, F. Hagras, H., and Callaghan, V. (2005) An Intelligent Fuzzy Agent Approach for Realising Ambient Intelligence in Intelligent Inhabited Environments, IEEE Transactions on System, Man and Cybernetics, Part A: Systems and Humans, 35, $1,55-65$.

Ducatel, K. Bogdanowicz, M. Scapolo, F. Leijten, J., Burgelman, J. (2003) Ambient intelligence: From vision to reality. In: Riva, G. Vatalaro, F. Davide, F. Alcaniz, M. (eds) Ambient Intelligence: The Evolution of Technology Communication and Cognation Towards the Future of Human-Computer Interaction (Volume 6 Emerging Communication: Studies in New Technologies and Practices in Communication), IOS Press.

Gustafsson, L. et al. (1995) Early clinical manifestations and the course of Alzheimer's disease related to regional cerebral blood flow and neuropathology. In: lqbal, K. Mortimer, J. A. Winblad, B., and Wisniewski, H. M. (eds), Research Advances in Alzheimer's Disease and Related Disorders. Wiley-Blackwell, Chichester, UK, pp. 209-218.

Hayes, T. L. Hunt, J. M. Adami, A., and Kaye, J. A. (2006) An Electronic Pillbox for Continuous Monitoring of Medication Adherence", In Proceedings of the 28th IEEE EMBS Annual International Conference, New York City, USA.

Matic, A. Mehta, P. Rehg, J. M. Osmani, V., and Mayora, O. (2008) Monitoring Dressing Activity Failures through RFID and Video, Methods of Information in Medicine, 47, 3, 229-234.

Mendel, J. (2001) Uncertain Rule-Based Fuzzy Logic Systems: Introduction and New Directions, Prentice Hall PTR 
Mihailidis, A. Carmichael, B., and Boger, J. (2004) The Use of Computer Vision in an Intelligent Environment to Support Aging-in-Place, Safety, and Independence in the Home, IEEE Transactions on Information Technology in Biomedicine, 8, 3, 238-247.

Mynatt, E. D. Melenhorst, A. S, Fisk, A. D, Rogers, W. A. (2004) Aware Technologies for Aging in Place: Understanding User Needs and Attitudes, Pervasive Computing, 3, 2, 36-41.

[29] Neergaard, L. (2007) Can Motion Sensors Predict Dementia? The Associated Press, Tuesday, $19^{\text {th }}$ June.

PDE-3193 (2004) Planning Guide for Dementia Care at Home: A Reference Tool for Care Managers, Alzheimer's Association South Central Wisconsin Chapter et al, Wisconsin, USA.

OSGi (2011) OSGi Alliance. http://www.osgi.org.
Peters, C. Wachsmuth, S., and Hoey, J (2009) Learning to Recognise Behaviours of Persons with Dementia using Multiple Cues in an HMM-Based Approach, In Proceedings of the $2^{\text {nd }}$ International Conference on Pervasive Technologies Related to Assistive Environments, Corfu, Greece, 23-25 June.

PureLink Technology (2011) Technologies. http://www.purelink.ca/Technologies/RelatedTechn ologies.aspx\#sensor, June 2010.

Wang, L. X. (2003) The MW Method Completed: A Flexible System Approach to Data Mining," IEEE Transactions on Fuzzy Systems, 11, 6, 768-782.

Woolham, J. Gibson, G., and Clark, P. (2007) Assistive Technology, Telecare, and Dementia: Some Implications of Current Policies and Guidance, Research Policy and Planning 24, 3, 149-164. 